\title{
Nexo Epidemiológico do Câncer Relacionado ao Trabalho no Município de Londrina-PR
}

doi: https://doi.org/10.32635/2176-9745.RBC.2021v67n3.1328

\author{
Epidemiological Nexus of Work-Related Cancer in the City of Londrina-PR \\ Nexo Epidemiológico del Cáncer Laboral en la Ciudad de Londrina-PR
}

\author{
Renata Cristina Silva Baldo'; Claudete Stábile Ribeiro Romaniszen²; Regina Stella Spagnuolo³; Fátima Sueli Neto Ribeiro4; Ildeberto \\ Muniz de Almeida ${ }^{5}$
}

\section{RESUMO}

Introdução: O câncer relacionado ao trabalho é um agravo de notificação compulsória desde 2004 no Sistema Único de Saúde, no entanto, os serviços de saúde náo incorporaram essa prática em suas rotinas de trabalho. Objetivo: Descrever o processo de estabelecimento do nexo epidemiológico do câncer relacionado ao trabalho em um hospital de referência no município de Londrina-PR, desde a seleção do caso até a notificação em sistema nacional de agravos de notificação. Método: Trata-se de estudo de abordagem qualitativa, descritiva, cuja coleta de dados se deu por meio de registro do histórico ocupacional. Identificou-se o nexo epidemiológico quando foi possível atender aos critérios de temporalidade, plausibilidade biológica e coerência bibliográfica entre a atividade ocupacional e o tipo de câncer diagnosticado. Resultados: Foram realizadas 579 anamneses e, destas, em 305, foi possível estabelecer nexo epidemiológico entre o câncer e o trabalho, sucedendo à digitaçáo em banco de dados do Sistema de Informação de Agravos de Notificação. Conclusáo: O estudo apresenta uma metodologia que possibilita a identificação e a notificação dos casos e que pode ser reproduzida em outros serviços de saúde, a fim de dar visibilidade ao câncer relacionado ao trabalho.

Palavras-chave: Saúde do Trabalhador; Câncer Ocupacional; Sistemas de Informação em Saúde; Notificação de Doenças.

\section{ABSTRACT}

Introduction: Work-related cancer has been a condition of compulsory notification since 2004 in the Unified Health System, however, health services have not incorporated this practice into their working routines. Objective: To describe the process of establishing the epidemiological nexus of work-related cancer in a reference hospital in the city of Londrina-PR, from the selection of the case to the notification in the national system of notifiable diseases. Method: Qualitative, descriptive approach study, whose data was collected through the record of occupational history. The epidemiological nexus was identified when it was possible to meet the criteria of temporality, biological plausibility and bibliographic coherence between occupational activity and the type of cancer diagnosed. Results: 579 anamneses were carried out, and of these, in 305 it was possible to establish an epidemiological nexus between cancer and work, after keying in the database of the Notifiable Diseases Information System. Conclusion: The study presents a methodology that allows the identification and notification of cases and that can be reproduced in other health services, in order to give visibility to work-related cancer.

Key words: Occupational Health; Occupational Cancer; Health Information Systems; Disease Notification.

\section{RESUMEN}

Introducción: El cáncer relacionado con el trabajo es condición de notificación obligatoria desde 2004, en el Sistema Único de Salud, sin embargo, los servicios de salud no han incorporado esta práctica en sus rutinas laborales. Objetivo: Describir el proceso de establecimiento del nexo epidemiológico del cáncer relacionado con el trabajo en un hospital de referencia de la ciudad de Londrina-PR, desde la selección del caso hasta la notificación en el sistema nacional de enfermedades de declaración obligatoria. Método: Se trata de un estudio con enfoque cualitativo, descriptivo, cuyos datos fueron recolectados mediante el registro de antecedentes laborales. El vínculo epidemiológico se identificó cuando fue posible cumplir con los criterios de temporalidad, plausibilidad biológica y coherencia bibliográfica entre la actividad ocupacional y el tipo de cáncer diagnosticado. Resultados: Se realizaron 579 anamnésicos, de los cuales en 305 se logró establecer un vínculo epidemiológico entre el cáncer y el trabajo, logrando la tipificación en la base de datos del Sistema de Información de Enfermedades Notificables. Conclusión: El estudio presenta una metodología que permite la identificación y notificación de casos y que puede ser reproducida en otros servicios de salud, con el fin de dar visibilidad al cáncer relacionado con el trabajo.

Palabras clave: Salud Laboral; Cáncer Profesional; Sistemas de Información en Salud; Notificación de Enfermedades.

\footnotetext{
1,2Secretaria Municipal de Saúde de Londrina/Núcleo de Atenção à Saúde do Trabalhador. Londrina (PR), Brasil. E-mails: renatabaldo@sercomtel.com.br; luizclau@sercomtel.com.br. Orcid iD: https://orcid.org/0000-0003-0355-4722; Orcid iD: https://orcid.org/0000-0001-9723-1259

3,5Universidade Estadual Paulista (Unesp)/Faculdade de Medicina de Botucatu. Botucatu (SP), Brasil. E-mails: rstella10@yahoo.com.br; ildeberto.almeida@unesp.br. Orcid iD: https://orcid.org/0000-0002-6977-4165; Orcid iD: https://orcid.org/0000-0002-8475-3805

${ }^{4}$ Universidade do Estado do Rio de Janeiro (Uerj). Rio de Janeiro (RJ), Brasil. E-mail: fatsuerj@gmail.com. Orcid iD: https://orcid.org/0000-0002-8201-4806

Endereço para correspondência: Renata Cristina Silva Baldo. Rua Ernani Lacerda de Thayde, 45 - Gleba Palhano. Londrina (PR), Brasil. CEP 86055-630.

E-mail: renatabaldo@sercomtel.com.br
} 


\section{INTRODUÇÃO}

O Instituto Nacional de Câncer José Alencar Gomes da Silva (INCA) ${ }^{1}$ estimou a ocorrência de 625 mil casos novos de câncer no Brasil para cada ano do triênio 2020-2022, sendo o câncer de pele não melanoma o mais incidente na populaçáo brasileira (176.940 mil casos novos), seguido pelo de mama feminina $(66.280 \mathrm{mil})$, próstata $(65.840$ mil), colón e reto (41 mil), pulmão (30 mil), útero (colo e corpo, 23 mil) e estômago (21 mil).

Estima-se que $80 \%$ dos casos de câncer estão relacionados a fatores ambientais em maior ou menor grau evitáveis. Esses fatores envolvem água, terra, ar, ambiente de consumo (alimentos, medicamentos, fumo, álcool e produtos domésticos), ambiente cultural (costumes e hábitos de vida) e ambiente ocupacional2 ${ }^{2}$.

A proporção de casos de câncer atribuída a exposições ocupacionais oscila entre $4 \%$ e $40 \%$, dependendo do tipo de tumor e metodologia empregada ${ }^{2}$. Por sua vez, a mensuração da exposição a agentes cancerígenos em ambientes de trabalho é uma tarefa que requer instrumental e metodologia própria pouco aplicada de forma confiável no Brasil.

O Ministério da Saúde reconhece alguns tipos de cânceres relacionados ao trabalho pela Portaria no ${ }^{\circ} .1 .339$ de $1999^{3}$. Em 2014, a Portaria Interministerial no. 9 de $2014^{4}$ assinada pelos Ministérios da Saúde, do Trabalho e da Previdência reconhecem uma Lista Nacional de Agentes Cancerígenos para Humanos (LINACH) e, entre estes, os presentes no ambiente de trabalho ${ }^{4}$.

No ano de 2004, o Ministério da Saúde publicou a Portaria no 777 de 28 de abril de $2004^{5}$ que tornou obrigatória a notificaçáo dos agravos relacionados ao trabalho, entre eles, o câncer. Esse documento apresentou outras versôes, sendo a mais atual a Portaria $n^{\circ} .205$ de 17 de fevereiro de $2016^{6}$.

$\mathrm{O}$ instrumento para essa notificação é a ficha do Sistema Nacional de Agravos de Notificação (SINAN). Ressalta-se que o Brasil é o único país do mundo a tornar obrigatória a notificaçáo desse agravo em serviços de saúde. Apesar da obrigatoriedade, até o ano de 2018, havia apenas 1.644 notificações registradas no SINAN 7 .

Neste contexto, em 2012, o Ministério da Saúde publicou as Diretrizes para a Vigilância do Câncer Relacionado ao Trabalho (DVCRT) ${ }^{2}$. Essa Diretriz oferece informaçôes técnicas e epidemiológicas para que sejam averiguadas, na história profissional do trabalhador, informaçóes referentes a contatos com compostos potencialmente cancerígenos presentes no ambiente de trabalho ${ }^{2}$.

Cabe salientar que o câncer, por ser uma doença com período de latência prolongado, requer ferramentas que recuperem as informaçôes sobre as experiências laborais do indivíduo, muitas vezes, em um passado distante ao do diagnóstico da doença, e minuciosa interpretação retrospectiva dessa possível exposição $0^{8-10}$. Essa dificuldade já era apontada por Acheson ${ }^{8}$ na década de 1960.

Em Londrina, no período de janeiro a dezembro de 2005, um estudo identificou, entre 784 pacientes atendidos no Hospital de Câncer, 296 (37,75\%) com diagnóstico de câncer constante na lista de neoplasias relacionadas ao trabalho $(\text { LNRT })^{11}$. A experiência do município de Londrina com o tema vem de longa data, motivo pelo qual o INCA o elegeu como município-piloto para validação das DVCRT. Desde então, o município vem realizando capacitaçôes em parceria com o Grupo de Ensino e Pesquisa em Epidemiologia do Câncer (Gepec) da Universidade do Estado do Rio de Janeiro (Uerj) por diversos Estados e, em um trabalho conjunto, aprimorando o processo de notificação.

Apesar do caráter compulsório da notificação do câncer relacionado ao trabalho desde 2004, no Sistema Único de Saúde (SUS), apenas em 2012 a rede SUS pôde contar com as Diretrizes para Notificação do Câncer Relacionado ao Trabalho.

Todavia, os serviços de saúde não incorporaram essa prática nas suas rotinas de trabalho, justificando-se, assim, o desenvolvimento deste estudo, que não buscou identificar, entre os casos totais de um determinado tipo de câncer na população geral, os que tiveram exposição ocupacional, mas sim estabelecer a práxis para o estabelecimento do nexo epidemiológico e posterior notificação em um serviço de saúde.

Diante desse cenário, questiona-se Como o Núcleo de Atenção à Saúde do Trabalhador (NAST) do município de Londrina-PR concede os nexos epidemiológicos de câncer relacionado ao trabalho, a fim de dar visibilidade aos casos por meio da notificação no SINAN?

Desta forma, objetivou-se descrever o processo de estabelecimento do nexo epidemiológico dos casos de câncer relacionado ao trabalho a partir de um hospital de referência no município de Londrina-PR.

\section{MÉTODO}

Trata-se de um estudo de abordagem qualitativa, descritiva, que apresenta o processo de estabelecimento do nexo epidemiológico dos casos de câncer relacionado ao trabalho em um hospital de referência no município de Londrina-PR. O trabalho faz parte de um recorte de doutorado intitulado "Implantação da vigilância do câncer relacionado ao trabalho em Londrina realizado entre 2011 e 2014" cujo método foi a pesquisa-ação ${ }^{12}$.

Teve como cenário um Hospital classificado como Centro de Assistência de Alta Complexidade em 
Oncologia (Cacon), por atender aproximadamente 90,0\% dos casos de cânceres do município e Região Metropolitana.

A eleição, análise e avaliação dos casos foram realizadas por dois técnicos qualificados em avaliação da exposição ocupacional e atuantes no serviço de saúde do trabalhador do município de Londrina.

$\mathrm{O}$ processo de seleção se deu in loco utilizando-se de amostra intencional; ou seja, aquela em que os pesquisadores escolhem os participantes segundo o objeto de estudo.

A coleta de dados ocorreu entre abril de 2013 e março de 2020 e elegeu como participantes pacientes com diagnósticos de câncer confirmados por médico-assistente e constantes na DVCRT, internados nas unidades de tratamento clínico ou cirúrgico, estarem conscientes e comunicativos ou terem um familiar que pudesse fornecer as informaçóes necessárias para a anamnese ocupacional, perfazendo um total de 579 .

Os instrumentos de coleta de dados foram as informaçóes do prontuário eletrônico por meio das variáveis: nome do paciente, leito, número do prontuário, tipo e data de diagnóstico do tumor primário e ficha de anamnese proposta nas DVCRT, contendo espaço para o recordatório do histórico ocupacional. Estas foram testadas em pacientes internados em outro hospital do município e adaptadas pelos técnicos, a fim de otimizar o processo.

Tal ficha consiste em uma folha (frente e verso), na qual a primeira página contém os dados de identificação do paciente e espaço para o recordatório do histórico ocupacional. No verso da folha, foram criados campos para sistematizar essas informaçóes, visando a favorecer a identificação da ocupação, o agente e o tempo de exposição de cada atividade descrita.

A anamnese durou em média 20 minutos, foi aplicada a todos os pacientes elegíveis e adotou como referencial teórico para subsidiar a relação de nexo com a ocupação as $\mathrm{DVCRT}^{2}$, além de informaçôes técnicas sobre cada processo de trabalho ${ }^{13}$.

Os participantes assinaram o Termo de Consentimento Livre e Esclarecido (TCLE) após serem esclarecidos sobre a pesquisa. Este estudo foi aprovado pelo Comitê de Ética da Universidade Norte do Paraná sob o número CAAE: 07593612.8.0000.0108. Respeitaram-se todas as normas da Resolução no ${ }^{\circ}$ 466/2012 do Conselho Nacional de Saúde ${ }^{14}$.

A pesquisa atendeu às orientaçóes dos critérios consolidados para relatar uma pesquisa qualitativa (do inglês, Consolidated criteria for reporting qualitative research - Coreq ${ }^{15}$ ), um guia com 32 itens que apoiam o desenvolvimento de estudos qualitativos.

\section{RESULTADOS E DISCUSSÃO}

Foram realizadas 579 anamneses. Em 305 delas, foi possível estabelecer nexo epidemiológico entre o câncer e o trabalho. Para descrever o processo, os resultados serão apresentados em quatro etapas: escolha dos participantes; descrição da anamnese ocupacional; análise e estabelecimento do nexo epidemiológico, sendo este objeto central deste estudo; e notificaçôes realizadas no banco de dados do SINAN (Figura 1).

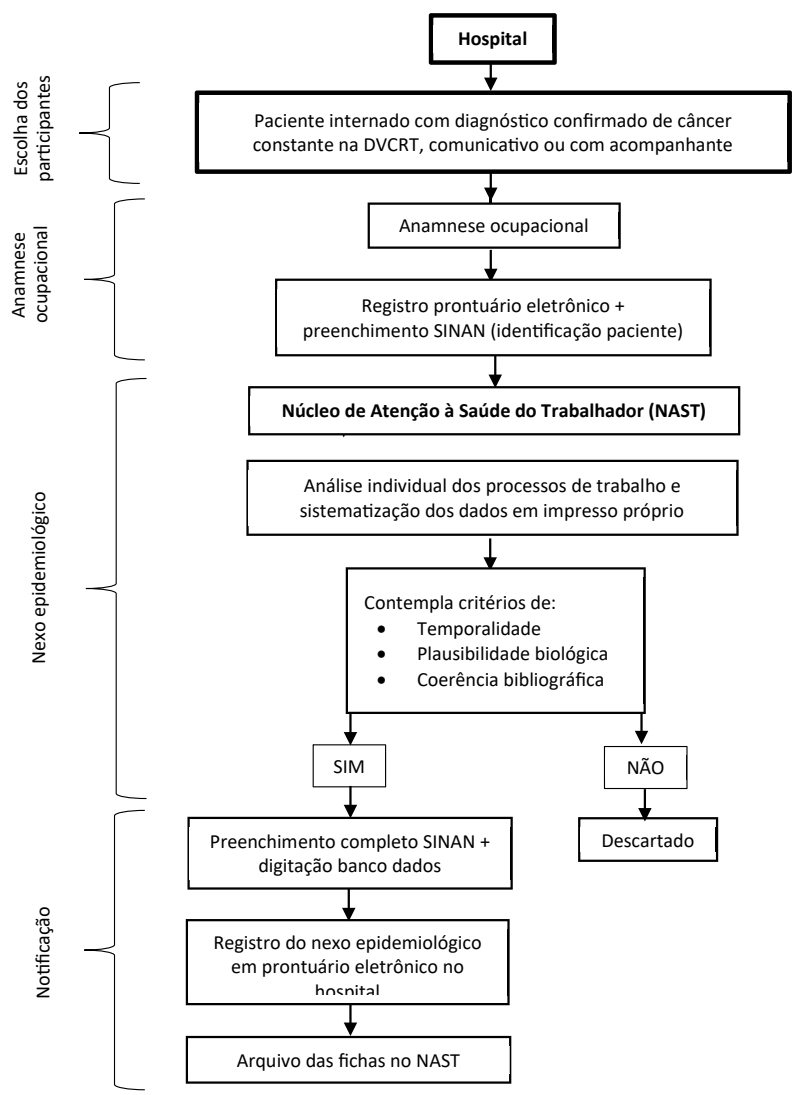

Figura 1. Fluxograma das etapas para o estabelecimento do nexo epidemiológico do câncer relacionado ao trabalho e notificação no SINAN

\section{ESCOLHA DOS PARTICIPANTES}

Dos muitos tipos de câncer constantes nas DVCRT, a eleiçẫo de alguns seguiu a priorização que se deu ao longo do curso de capacitação ocorrido no município em 2011. Neste, foram consensuados alguns tipos, baseados no perfil produtivo do município, na literatura sobre o objeto de estudo e na facilidade de acesso aos pacientes internados em hospitais de referência para câncer ${ }^{16}$.

Desta forma, começou-se pelos casos de cânceres de cabeça e pescoço e bexiga. A partir do mês de novembro de 2013, as buscas foram ampliadas para os pacientes com os outros tipos de cânceres constantes na $\mathrm{DVCRT}^{2}$. 
Iniciou-se com a obtençấo da lista dos pacientes nas unidades de internação clínica e cirúrgica por meio do sistema de prontuário eletrônico, a fim de identificar os casos com diagnósticos confirmados de cânceres, cujo tumor primário constasse na DVCRT ${ }^{2}$. Esse diagnóstico encontra-se nos laudos de exames e na evoluçáo do médico-assistente.

Em seguida, foram transcritos para o instrumento de anamnese ocupacional o nome do paciente, o leito, o tipo e a data de diagnóstico do tumor primário.

\section{ANAMNESE OCUPACIONAL}

Nesta etapa, entrevistaram-se os pacientes individualmente, iniciando pela ocupação mais antiga (aquela em que o trabalhador começou suas atividades laborais levando em conta a idade, lugar e em qual atividade se deu esse início). $\mathrm{Na}$ descrição, foram relatadas todas as atividades informadas pelo paciente em cada um dos locais de trabalho, independentemente de ter ou não vínculo empregatício. Ainda, os pacientes foram interrogados sobre os agentes/substâncias possivelmente utilizados em cada atividade descrita. A importância desse momento "face-a-face" para detalhamento de um histórico ocupacional foi observada em estudo realizado na França9.

A anamnese ocupacional constitui-se um dos principais instrumentos para a investigação das relaçóes saúde-trabalho-doença, favorecendo o diagnóstico correto do agravo e sua etiologia com o trabalho ${ }^{8,13}$. Embora historicamente a anamnese ocupacional esteja associada ao profissional médico ${ }^{13}$, em Londrina-PR observou-se que a responsabilidade da sua realizaçáo não deve se restringir apenas a esse profissional, visto que ela não tem um caráter diagnóstico. Assim, todos os profissionais de saúde devidamente capacitados e envolvidos com a temática podem realizar a referida investigação ocupacional ${ }^{17}$.

Em seguida, foi realizado o registro na evoluçáo do paciente, indicando que a anamnese ocupacional foi concluída, assim como o preenchimento inicial da ficha do SINAN no que se refere à identificação do paciente.

Nessa identificação, consta o campo "ocupação" preenchido pelo profissional do registro no momento da internaçáo, porém, para a realização do nexo epidemiológico, a ocupação a ser considerada é aquela que coincide com a exposiçấo a possíveis agentes cancerígenos. Neste estudo, dos 305 nexos epidemiológicos concedidos, em 122 (40,0\%), as ocupaçōes apontadas pelo Registro Hospitalar de Câncer (RHC) e as ocupaçóes compatíveis com os nexos não coincidiram.

O período entre a exposiçáo e a doença pode estender-se ao longo de décadas, e as informaçôes colhidas na anamnese ocupacional suplantam o preenchimento do campo "ocupação" nos registros de RHC. Desde a década de 1960, Acheson ${ }^{8}$ já apontava que a ocupação atual é de valor limitado, tendo em vista as mudanças que ocorrem ao longo da vida do trabalhador, o que de fato também foi observado na experiência de Londrina.

Portanto, este estudo mostrou que não é possível estabelecer o nexo epidemiológico para fins de notificação no SINAN apenas comparando a ocupação atual referida pelo paciente com a LNRT. Em muitos casos, por exemplo, o paciente pode se declarar "porteiro", quando na verdade atuou como lavrador na maior parte da vida laboral.

\section{ESTABELECIMENTO DO NEXO EPIDEMIOLÓGICO}

Com as informaçôes ocupacionais e o diagnóstico do câncer primário, procurou-se detalhar cada processo de trabalho descrito pelo paciente durante suas atividades laborais, a fim de identificar uma possível exposição a agentes cancerígenos. Em seguida, foram sistematizadas todas as atividades relatadas de tal forma que, ao final, foi possível identificar a ocupação, o agente e o tempo de exposição, permitindo verificar se os achados subsidiaram ou não a relação da ocupação com o agente/exposição e o câncer em questáo.

De acordo com a literatura, a relaçáo entre a exposição e o tipo de câncer capaz de configurar um nexo epidemiológico deve basear-se em argumentos que levam à sua presunção ${ }^{13,18}$. Portanto, a convicçâao técnica deve ser plena, pois pouco se conhece sobre as possibilidades das exposições cruzadas funcionando como agente causal ou fator contributivo para diversos tipos de câncer.

Além disso, a exemplo do que se dá no raciocínio diagnóstico de agravos multicausais, em que o conhecimento existente náo permite nem prova cabal da existência e nem da ausência de relaçáo com o trabalho, a conclusão passa a se basear no cotejamento de achados existentes em favor e contra o nexo.

Nas situaçôes mais simples, pró-nexo, estão identificados indícios de: a) exposição de risco para o agravo; b) boas condiçôes de saúde no início da exposição; c) evolução com instalação do agravo em conformidade com tempo de latência já descrito na literatura e outros achados favoráveis ao reconhecimento da natureza secundária do agravo, como por exemplo, acometer trabalhador em idade inferior àquela em que o agravo costuma aparecer com origem idiopática; d) afastamento de causas não ocupacionais do mesmo agravo.

Para a possível associação entre exposiçấo e doença, os aspectos fundamentais considerados foram: temporalidade, plausibilidade biológica e coerência bibliográfica ${ }^{2}$.

Alguns autores relatam que uma das dificuldades encontradas para estabelecimento do nexo é em relação à temporalidade, dada a variabilidade dos períodos de 
latência referidos para o desenvolvimento do câncer ${ }^{8,9,18-21}$. Entende-se por latência o tempo entre a exposição a um determinado agente e o início presumido da doença ${ }^{2}$.

Para este estudo, utilizou-se, como referência para determinar o período de latência mínima entre a primeira exposição ao agente supostamente cancerígeno e o diagnóstico da doença, 20 anos para tumores sólidos e cinco anos para tumores hematológicos, conforme aponta as DVCRT $^{2}$.

No que diz respeito à plausibilidade biológica, foram levados em conta os agentes/substâncias referidos na anamnese ocupacional e suas respectivas evidências de carcinogenicidade para humanos estabelecidos pela DVCRT $^{2}$. Cabe lembrar a importância da observação das vias de exposição, os possíveis equipamentos que impedem o contato humano e/ou o enclausuramento do processo de trabalho.

No que se refere à coerência bibliográfica, utilizaram-se, além das DVCRT, outras bibliografias pertinentes ao tema que se fizeram necessárias para subsidiar a análise dos processos de trabalho, como por exemplo, livros de química orgânica, citologia, oncologia, sites relacionados a composição/uso de agrotóxicos de tintas, solventes, combustíveis, poeiras, radiações, entre outros. A exposição no ambiente de trabalho é tão rica como singular; assim, esse critério não pode ser definitivo para excluir a presunção do nexo. Em muitos casos, algumas substâncias sáo utilizadas na manutenção, e o reconhecimento do risco no processo de trabalho é subestimado.

Baseado nos três critérios, foi estabelecido nexo epidemiológico em 305 pacientes (52,7\%) das 579 anamneses realizadas. A distribuição do total das anamneses e nexos por tipo de tumor está apresentada no Gráfico 1.

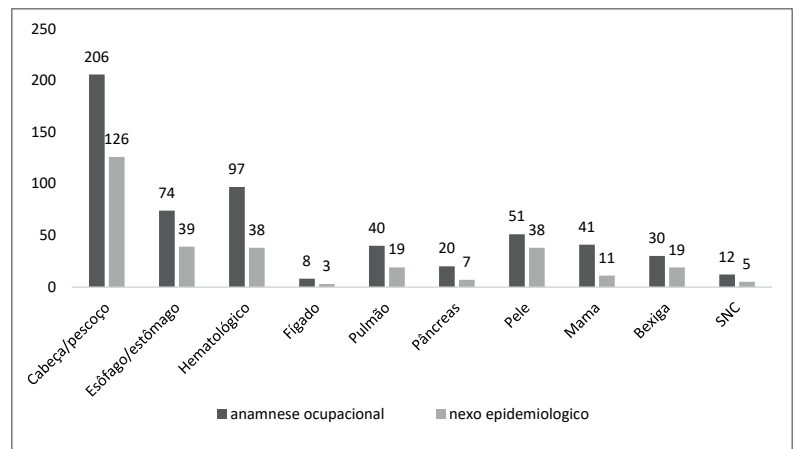

Gráfico 1. Distribuição das anamneses ocupacionais e nexo epidemiológico estabelecido segundo localização do tumor primário. Londrina, abril de 2013 a março 2020

Estudo de Doll e Peto ${ }^{22}$ refere que $4 \%$ dos casos de câncer são atribuídos à exposição ocupacional e Leigh ${ }^{23}$ atribui entre $8 \%$ e $16 \%$ a proporçáo de casos relacionados à exposição ocupacional. Não existe na literatura estudos assemelhados para população internada.
No entanto, diferente do que esses autores apresentam, este estudo não buscou identificar, entre os casos totais de um determinado tipo de câncer na população geral, os que tiveram exposição ocupacional. $\mathrm{O}$ intuito foi de determinar a práxis para o estabelecimento do nexo epidemiológico e posterior notificação em um serviço de saúde.

O processo de identificar a relação entre a exposição e o tipo de câncer não está bem sistematizado nos procedimentos do SUS e a iniciativa neste sentido foi realizada pelos protocolos publicados pelo Ministério da $S_{\text {Saúde }}^{2}$ e por Ribeiro ${ }^{24}$. Neste caso, a operacionalização por meio de um Cacon, para identificaçáo dos casos e o estabelecimento do nexo por profissional da área de Saúde do Trabalhador, demonstra a importância e a possibilidade de integração intraSUS, que muito se apregoa, mas com dificuldade para ser realizada.

\section{NOTIFICAÇÃO NO SINAN}

Esta última etapa consistiu em transcrever para a ficha do SINAN as informaçóes elencadas que embasaram o nexo epidemiológico e na digitação em banco de dados nacional.

O SINAN deve ser preenchido pela fonte notificadora e encaminhado posteriormente para digitação, quer no serviço de vigilância epidemiológica do município, quer em outro local onde é possível proceder à digitação. Em londrina, a digitação é realizada pelos pesquisadores que também atuam no NAST.

Após a notificação, os pesquisadores retornam ao hospital para registrarem em prontuário eletrônico os dados referentes ao nexo estabelecido. Dessa forma, a informação fica disponível para visualização pela equipe de saúde do hospital e, ao final, a ficha em papel é arquivada no próprio NAST.

No Brasil, a notificação do câncer relacionado ao trabalho é compulsória desde 2004 pela Portaria no . $777^{5}$. O aumento da notificação no país é mais relevante a partir de 2011. Entre 2011 e 2018, foram registrados 1.571 casos de câncer relacionado ao trabalho no Brasil, sendo 596 (43,4\%) no Paraná7. Em 2013, o município de Londrina iniciou a busca ativa para alimentar a notificaçâo dos casos no SINAN e, desde entáo, foram registrados 305 casos em Londrina.

A utilização do SINAN, em conjunto com os demais Sistemas de Informação em Saúde, consiste em uma importante ferramenta para facilitar a formulação e a avaliação das políticas, planos e programas de saúde, subsidiando o processo de tomada de decisóes, com vistas a contribuir para a melhoria da situaçáo de saúde da populaçãa 25 .

Países como França, Itália, República Checa, Eslováquia, Polônia e Canadá possuem registros de câncer ocupacional 
para reconhecimento previdenciário, indenizaçóes e desencadeamento de açôes de prevenção ${ }^{9,19,20,26}$. No entanto, como o Brasil é o único país a notificar o câncer relacionado ao trabalho em uma base de dados nacional de serviços de saúde, não foi possível a comparação com estudos assemelhados.

A relevância dessas notificações reside também na possibilidade de identificar casos na população informal, em trabalhos temporários e nas demais formas não regulamentadas de ocupação que são invisíveis ao sistema previdenciário, mas que constituem mais de $60 \%$ da ocupação dos brasileiros.

Podem ser consideradas limitaçóes do trabalho as dificuldades em abarcar todos os pacientes com diagnósticos de câncer internados no hospital naquele período, a complexidade de identificar as exposições pregressas e a falta de uma política que promova a integração entre as áreas do SUS.

\section{CONCLUSÃO}

As etapas necessárias para o estabelecimento do nexo epidemiológico foram apresentadas de forma direta e demonstram sua factibilidade, desde o acesso aos prontuários e realizaçáo da anamnese ocupacional até a notificação do câncer relacionado ao trabalho no SINAN. Essa viabilidade se faz importante em um momento que o debate desse tema ainda é incipiente.

Esses resultados atendem à demanda científica de subsídio técnico à prática do serviço de saúde. $\mathrm{O}$ ineditismo da experiência relaciona-se mais fortemente com iniciativas pessoais de uma política local e termina por demonstrar as possibilidades de articulação entre serviços de área distintas na saúde, assistência ao câncer e saúde do trabalhador, sem custo adicional para impactar as notificaçōes no SUS e possível de ser replicado em outros cenários semelhantes.

\section{CONTRIBUIÇÕES}

Renata Cristina Silva Baldo contribuiu no delineamento do estudo, coleta, análise e interpretação dos dados, elaboração do manuscrito e revisão crítica. Claudete Stábile Ribeiro Romaniszen contribuiu na coleta, análise e interpretação dos dados, elaboração do manuscrito e revisão crítica. Regina Stella Spagnuolo contribuiu na elaboração do manuscrito e revisão crítica. Fátima Sueli Neto Ribeiro e Ildeberto Muniz de Almeida contribuíram na revisão crítica. Todos os autores aprovaram a versão final a ser publicada.

\section{DECLARAÇÃO DE CONFLITO DE INTERESSES}

Nada a declarar.

\section{FONTES DE FINANCIAMENTO}

Não há.

\section{REFERÊNCIAS}

1. Instituto Nacional de Câncer José Alencar Gomes da Silva. Estimativa 2020: incidência de câncer no Brasil [Internet]. Rio de Janeiro: INCA; 2020 [acesso 2020 out 20]. Disponível em: https://www.inca.gov.br/sites/ ufu.sti.inca.local/files/media/document/estimativa-2020incidencia-de-cancer-no-brasil.pdf

2. Instituto Nacional de Câncer José Alencar Gomes da Silva. Diretrizes para a vigilância do câncer relacionado ao trabalho. 2. ed. rev. atual. Rio de Janeiro: INCA; 2013.

3. Ministério da Saúde (BR), Gabinete do Ministro. Portaria $\mathrm{n}^{\circ} 1339$, de 18 de novembro de 1999. Institui a Lista de Doenças relacionadas ao Trabalho, a ser adotada como referência dos agravos originados no processo de trabalho no Sistema Único de Saúde, para uso clínico e epidemiológico, constante no Anexo I desta Portaria. (Ementa elaborada pela CDI/MS). Diário Oficial da União. 1999 nov 19; Seçáo 1:21.

4. Ministério do Trabalho e Emprego (BR), Gabinete do Ministro. Portaria Interministerial no 9 , de 7 de outubro de 2014. Publica a Lista Nacional de Agentes Cancerígenos para Humanos (LINACH), como referência para formulaçâo de políticas públicas, na forma de anexo a esta Portaria. Diário Oficial da União. 2014 out 8; Seção 1:140.

5. Ministério da Saúde (BR), Gabinete do Ministro. Portaria no 777, de 28 de abril de 2004. Dispóe sobre os procedimentos técnicos para a notificação compulsória de agravos à saúde do trabalhador em rede de serviços sentinela específica, no Sistema Único de Saúde - SUS. (Ementa elaborada pela Biblioteca/MS). Diário Oficial da União. 2004 abr 29; Seção 1:37.

6. Ministério da Saúde (BR), Gabinete do Ministro. Portaria $\mathrm{n}^{\circ}$ 205, de 17 de fevereiro de 2016. Define a lista nacional de doenças e agravos, na forma do anexo, a serem monitorados por meio da estratégia de vigilância em unidades sentinelas e suas diretrizes. Diário Oficial da União. 2016 fev 18; Seção 1:24.

7. SINAN: Sistema de Informação de Agravos de Notificação [Internet]. Salvador, BA: Centro Colaborador da Vigilância aos Agravos à Saúde do Trabalhador (ISCUFBA/CGSAY-MS). [data desconhecida] - [acesso 2020 ago 10]. Disponível em: http://www.ccvisat.ufba.br/ bases-de-dados/sinan

8. Acheson ED. Record linkage techniques in studies of the aetiology of cancer. Proc R Soc Med [Internet]. 1968 Jul [cited 2020 Oct 16];61(7):726-30. Available from: https://journals.sagepub.com/doi/ pdf/10.1177/003591576806100752 
9. Aubrun JC, Binet S, Bozec C, et al. Occupational cancer in France: epidemiology, toxicology, prevention, and compensation. Environ Health Perspect. 1999;107(Suppl 2):245-52. doi: https://doi.org/10.1289/ehp.99107s2245

10. Ribeiro FSN, Wünsch Filho V. Avaliação retrospectiva da exposição ocupacional a cancerígenos: abordagem epidemiológica e aplicação em vigilância em saúde. Cad Saúde Pública. 2004;20(4):881-90. doi: https://doi. org/10.1590/S0102-311X2004000400002

11. Romaniszen CSR. Neoplasias relacionadas ao trabalho: estudo de morbidade em um hospital de referência [monografia]. Londrina: Universidade Estadual de Londrina; 2008.

12. Thiollent M. Metodologia da pesquisa-ação. 18. ed. São Paulo: Cortez; 2011.

13. Ministério da Saúde (BR); Organização Pan-Americana da Saúde (BR). Doenças relacionadas ao trabalho: manual de procedimentos para os serviços de saúde. Brasília, DF: Ministério da Saúde, OPAS; 2001. (Série A. Normas e Manuais Técnicos, n. 114).

14. Conselho Nacional de Saúde (BR). Resolução no 466, de 12 de dezembro de 2012. Aprova as diretrizes e normas regulamentadoras de pesquisas envolvendo seres humanos. Diário Oficial da Uniâo. 2013 jun 13; Seção 1:59.

15. Tong A, Sainsbury P, Craig J. Consolidated criteria for reporting qualitative research (COREQ): a 32-item checklist for interviews and focus groups. Int J Qual Health Care. 2007;19(6):349-57. doi: https://doi. org/10.1093/intqhc/mzm042

16. Baldo RCS, Romaniszen CSR, Ribeiro FSN, et al. Eleição de prioridades para a vigilância do câncer relacionado ao trabalho no município de Londrina - Paraná, Brasil. Rev Bras Cancerol. 2014;60(3):215-222. doi: https://doi. org/10.32635/2176-9745.RBC.2014v60n3.466

17. Ross JB. Prevention of occupational disease: problems of data collection for adequate surveillance. Can Med Assoc J.1992;147(10):1443-5.

18. Chovil AC, McCracken WJ, Dowd EC, et al. Occupational cancer: experience in Ontario. Can Med Assoc J.1981;125(11):1237-41.

19. Merler E, Vineis P, Alhaique D, et al. Occupational cancer in Italy. Environ Health Perspect. 1999;107(Suppl 2):259-71. doi: https://doi.org/10.1289/ehp.99107s2259

20. Fabiánová E, Szeszenia-Dabrowska N, Kjaerheim K, et al. Occupational cancer in Central European Countries. Environ Health Perspect. 1999;107(Suppl 2):279-82. doi: https://doi.org/10.1289/ehp.107-1566282

21. Howard JK. Minimum latency \& types or categories of cancer [Internet]. Washington: World Trade Center Health Program; 2012 Oct 17 [revised 2015 Jan 6; cited 2020 Oct 16]. [about 12 p.]. Available from: https://www.cdc.gov/wtc/pdfs/policies/ wtchpminlatcancer2014-11-07-508.pdf
22. Doll R, Peto R. The causes of cancer: quantitative estimates of avoidable risks of cancer in the United States today. JNCI. 1981;66(6):1192-1308. doi: https://doi. org/10.1093/jnci/66.6.1192

23. Leigh JP. Occupations, cigarette smoking, and lung cancer in the epidemiological follow-up to the NHANES I and the California Occupational Mortality study. Bull N Y Acad Med. 1996;73(2):370-97.

24. Ribeiro FSN. Tornando visível o câncer relacionado ao trabalho: uma proposta metodológica de vigilância. In: Silva Neto BR, organizador. Prevenção e promoção de saúde 11 [Internet]. Ponta Grossa, PR: Atena Editora; 2019. p. 114-140. doi: https://doi.org/10.22533/ at.ed.4341918129

25. Ministério da Saúde (BR). Secretaria de Vigilância em Saúde. Departamento de Vigilância Epidemiológica. Sistema de Informação de Agravos de Notificação Sinan: normas e rotinas [Internet]. 2. ed. Brasília, DF: Ministério da Saúde; 2007. (Série A. Normas e manuais técnicos). [acesso 2020 out 16]. Disponível em: chromeextension://efaidnbmnnnibpcajpcglclefindmkaj/viewer. html?pdfurl=http\%3A\%2F\%2Fportalsinan.saude.gov. br\%2Fimages $\% 2$ Fdocumentos $\% 2$ FAplicativos $\% 2 F s$ inan_net\%2FManual_Normas_e_Rotinas_2_edicao. pdf\&clen $=644041 \&$ chunk $=$ true

26. Teschke K, Barroetavena MC. Occupational cancer in Canada: what do we know? CMAJ. 1992;147(10):1501-7.

Recebido em 27/11/2020 Aprovado em 5/2/2021 